\title{
A framework for engaging stakeholders in solving real- world water resources management problems
}

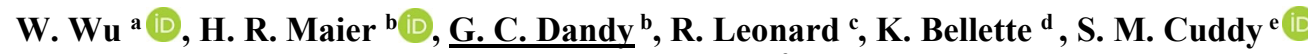 \\ and S. Maheepala ${ }^{f}$ \\ ${ }^{a}$ Department of Infrastructure Engineering, The University of Melbourne, Melbourne, VIC, ${ }^{b}$ School of Civil, \\ Environmental and Mining Engineering, The University of Adelaide, Adelaide, SA, ${ }^{c}$ Translational Health \\ Research Institute, Western Sydney University, Sydney, NSW, ${ }^{d}$ Environment Protection Authority, Adelaide, \\ $S A{ }^{e}$ CSIRO Land and Water, Canberra, ACT, ${ }^{f}$ Department of Environment, Land, Water and Planning, \\ Melbourne, VIC. \\ Email: graeme.dandy@adelaide.edu.au
}

\begin{abstract}
Multi-objective evolutionary algorithms (MOEAs) are becoming increasingly popular for solving environmental and water resources optimisation problems. In the past, the focus of these studies has generally been on methodological issues related to the optimisation algorithm, while the incorporation of stakeholder preferences in the MOEA solution process has largely been ignored. In recent years, there has been increased recognition of the need to apply these approaches to real-world problems to facilitate the realisation of their full potential. However, in most of these studies, stakeholder input was only used to direct the optimisation search process or select the final optimal solution(s), while the contribution of stakeholder input to other important components of the problem solving process was not considered. The reason for this is that the full consideration of stakeholder input in solving environmental and water resources optimisation problems requires the development of a more holistic approach, which involves a range of additional challenges.
\end{abstract}

To address these challenges, a framework for including stakeholder input in real-world optimisation problems has been developed as part of the Optimal Water Resources Mix (OWRM) project initiated by the South Australian Government through the Goyder Institute for Water Research. The framework includes a conceptual framework (Figure 1) and a procedure for its implementation. The framework was applied to an urban water supply security study for Adelaide, South Australia. A summary of the framework and how it was implemented to identify optimal water sourcing options for the Adelaide case study is presented in this paper.

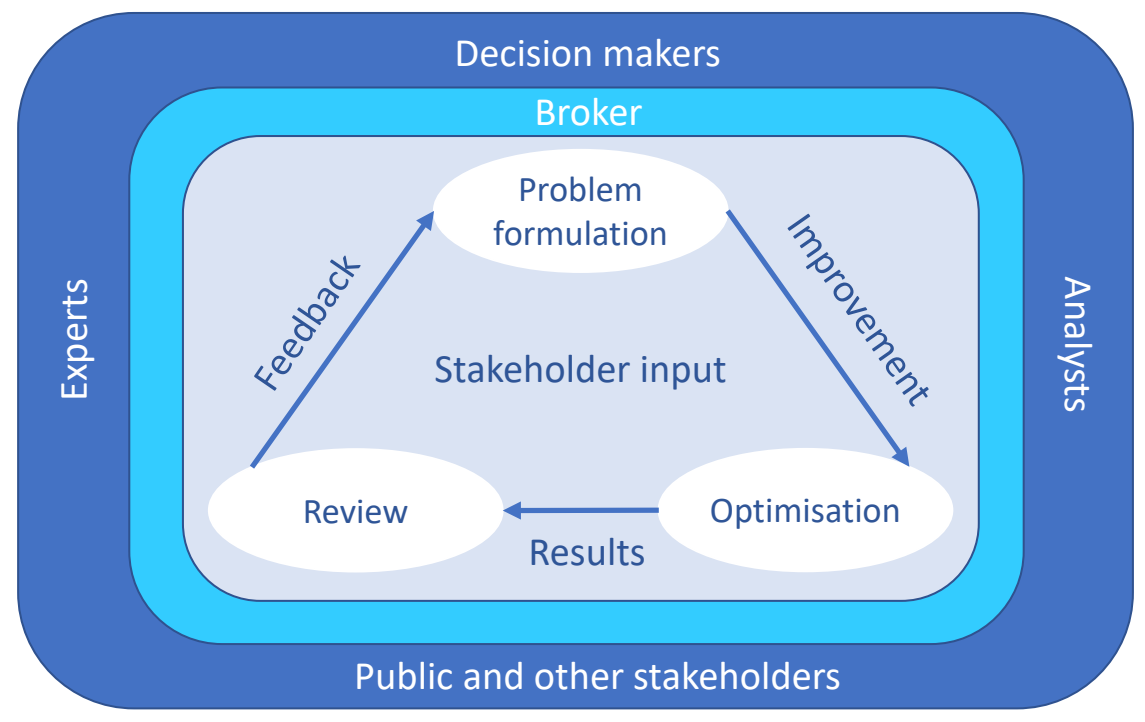

Figure 1. The proposed conceptual framework. Adapted from Wu et al. (2016)

This study highlights the important role of stakeholder input at the various stages of the problem formulation and optimisation process, analysis and results, although it can be expensive and time consuming to do so. It is recommended that adequate resources be made available for stakeholder engagement in project plans and budgets, as there needs to be clear and ongoing communication between stakeholder groups throughout the project. It also demonstrates that the use of MOEAs as the optimisation engine, together with appropriate stakeholder input, provides a combination that is well-suited to solving real-world water resources problems.

Keywords: Integrated urban water management, Multiobjective optimisation, Stakeholder preferences 


\section{INTRODUCTION}

Water resources management problems (e.g. Integrated Water Management) include multiple and often conflicting objectives. They can be solved using multi-objective evolutionary algorithms (MOEAs) linked to simulation models (Marques et al., 2015; Wu et al., 2017). While stakeholder input is commonly used in many simulation-based multi-objective decision support approaches (Black et al., 2014), the same does not apply to optimisation-based methods using MOEAs (Maier et al., 2019). This is mainly because the incorporation of stakeholder preferences in the MOEA solution process requires the development of a more holistic approach, which involves a range of additional challenges. These challenges include (1) formulating the optimisation problem to reflect the values of stakeholders, (2) translating the objectives of the optimisation process into a mathematical formulation, (3) striking a balance between representing stakeholder interests in the objectives and the ability to quantify them using appropriate models, (4) generating a diverse set of solutions that are acceptable from a practical perspective, (5) presenting the outputs in a form that is easily accessible and understandable to stakeholders, (6) providing opportunities for stakeholder feedback, and (7) accounting for practical constraints imposed by stakeholders (Wu et al., 2016).

Some progress has been made in the incorporation of stakeholder preferences in solving real-world problems using MOEA (e.g., Piemonti et al., 2013; Piscopo et al. 2015). However, in most of these studies, stakeholder input was only used to direct the optimisation process or select the final solution(s), while the contribution of stakeholder input to other important components of the problem solving process was not considered. In a study (Piscopo et al., 2015) where stakeholder input was considered to formulate problem constraints, only input from Analysts was considered.

In order to develop a holistic approach to real-life water resources optimisation problems where stakeholder input is considered, a framework has been developed (Wu et al, 2016). This framework is summarised in this paper. The application of the framework is demonstrated via a real-world integrated urban water management (IUWM) problem in Adelaide, South Australia.

\section{FRAMEWORK}

\subsection{Stakeholders and their roles}

Stakeholder input for formal optimisation of real-world problems is essential to ensure that the problem being solved meets stakeholder expectations and needs as much as possible and provides practical, useful, and acceptable solutions. Numerous typologies have been developed to describe approaches to stakeholder input (Reed, 2008) and types of stakeholders (Black et al., 2014). Methods have also been developed to make sure stakeholder input counteracts any power imbalance between the bureaucratic system and the lifeworld (i.e. people's everyday lived experience and the external viewpoint of the "systems") (Larson et al., 2010). Optimisation is usually a bureaucratic system driven, planner-centred process and the need for quantitative input to be collected from the public limits the scope for engaging with their lifeworlds. Together, these factors suggest a pragmatic, planner-centred approach with limited public engagement is most appropriate in the context considered here. Four categories of stakeholders have been suggested (Wu et al. 2016): Decision Makers (DMs), Analysts (including the Broker), Experts, and the Public and other stakeholders (POSs), whose roles are detailed below.

The DMs: They set the agenda and are responsible for making the final decision in relation to the project or policy that the optimisation process will inform. For public projects, they are usually government executives, board members and elected representatives who decide the problem to be solved and define the limits on solutions. The identification of the DMs as stakeholders means that the bureaucratic system is open to scrutiny, rather than being an unquestioned frame for a project.

The Analysts: They are the ones who conduct the project-related analysis. Their professional identity and reputation might be affected by the outcomes. Identifying the Analysts themselves as stakeholders recognises how the Analysts' choices may affect the outcomes.

The Broker: Often considered as part of the Analysts, the Broker has some pre-existing relationship with the DMs, but is independent from them. Therefore, the Broker can be a key figure in keeping open two-way communication between DMs, Experts, and Analysts.

The Experts: They have recognised scientific and management expertise related to the issue being addressed, as well as access to specialised knowledge or data that are needed to complete the task. The Analysts need to cultivate good relationships with the Experts and recognise their divergent views. Suitable respect for their expertise, the value of the information being provided, and the need for confidentiality, is often the key. 
The public and other stakeholders (POSs): They are the people who are affected by the outcomes of the project or are interested in the project, but are not included in the three categories (i.e. The Broker is part of the Analysts) listed above. Apart from the general public, other stakeholders might include the media, not-forprofit organisations operating in the field, and communities who may be particularly affected by any developments based on the technical solution adopted. They represent a large number of people who are likely to have very diverse and sometimes conflicting views. The engagement of this category of stakeholders needs to be actively managed and in the present research the method for engaging the public was approved by the CSIRO Social Science Human Research Ethics Committee.

\subsection{The conceptual framework}

The conceptual framework that includes stakeholder input in the optimisation of real-world problems is illustrated in Figure 1. The framework includes the stages commonly associated with solving optimisation problems, i.e. problem formulation and optimisation, and an additional review stage, where feedback from all stakeholders is sought and incorporated into the next iteration of problem formulation. As can be seen from Figure 1, stakeholder input is required at every stage of the process. However, the level and form of stakeholder input may vary depending on the role of different groups of stakeholders (i.e. Analysts, DMs, Experts and POSs) and the types of information required. Given the optimisation focus of the framework, the Analysts have significant and direct input at all steps of the process due to their active involvement in the entire process. They often construct the initial problem formulation and conduct the optimisation work based on information provided by the DMs and Experts. It is also the Analysts who present the optimisation results to DMs, Experts and POSs during the review process, seek feedback and incorporate this into the improved problem formulation. Because stakeholders have different perspectives, knowledge and experience to offer, significant benefit can arise from the engagement of an independent Broker to facilitate interaction and knowledge exchange between stakeholder groups. Due to potential changes in stakeholder thinking which often occur during problem solving and can result in changes to problem formulation, this framework is an interactive process.

\section{IMPLEMENTATION OF THE FRAMEWORK}

\subsection{Adelaide case study}

This study was part of the Optimal Water Resources Mix (OWRM) project initiated through the Goyder Institute for Water Research. The aim of the project was to develop and demonstrate an approach that could be used to identify the most cost-effective, environmentally sustainable and socially acceptable portfolio of water sources to meet the current (as at 2013) and future (2050) water demand of Adelaide. The geographic extent of the case study covers the Greater Adelaide area. The water sources considered include surface water from the Mount Lofty Ranges (MLR) and the River Murray (RM), the Adelaide desalination plant (ADP), harvested stormwater and reclaimed wastewater from Adelaide's 3 wastewater treatment plants (WWTPs). The project took approximately 1.5 years. Details of the case study are documented in Wu et al. (2016).

\subsection{Identification of stakeholders}

A summary of the stakeholders for the Adelaide case study, including the DMs, Analysts, Experts and POSs, is provided in Table 1. The principal DM in this project was the South Australian Government, as represented by staff from the Department for Environment, Water and Natural Resources (DEWNR). The Analysts included researchers from the University of Adelaide and the Commonwealth Scientific and Industrial Research Organisation (CSIRO), with expertise in simulation modelling, optimisation, stakeholder engagement and project management. The Experts included representatives from SA Water and the Environment Protection Authority (EPA) of South Australia, with expertise in water resource modelling and management, and environmental impact assessment; as well as a reference panel selected from key agencies based on technical expertise and/or policy interest. The Broker was from Flinders University who also had a background in water management in government. The POSs included a wider stakeholder group, which consisted of representatives from state and local government with water resources, urban planning and related portfolios, relevant non-government organisations (NGOs), as well as people with diverse relationships to water (e.g. the green perspective, such as water activists, community gardeners, and eco-village residents; high water users, such as parents of teenagers and swimming pool owners; businesses; renters; low income earners; and demographic diversity, including people from all adult age groups and 4 different ethnic backgrounds). 
Wu et al., A framework for engaging stakeholders in solving real-world water resources management problems

\subsection{Implementation process}

The implementation process of the framework is based on the systems approach (Biswas, 1976), which has been applied in a variety of application areas, such as policy analysis (Walker, 2000) and can be divided into 3 stages including 9 steps. The implementation of the framework for the Adelaide case study is presented below.

Table 1. Summary of stakeholders and their roles for Adelaide case study (Adapted from Wu et al. (2016))

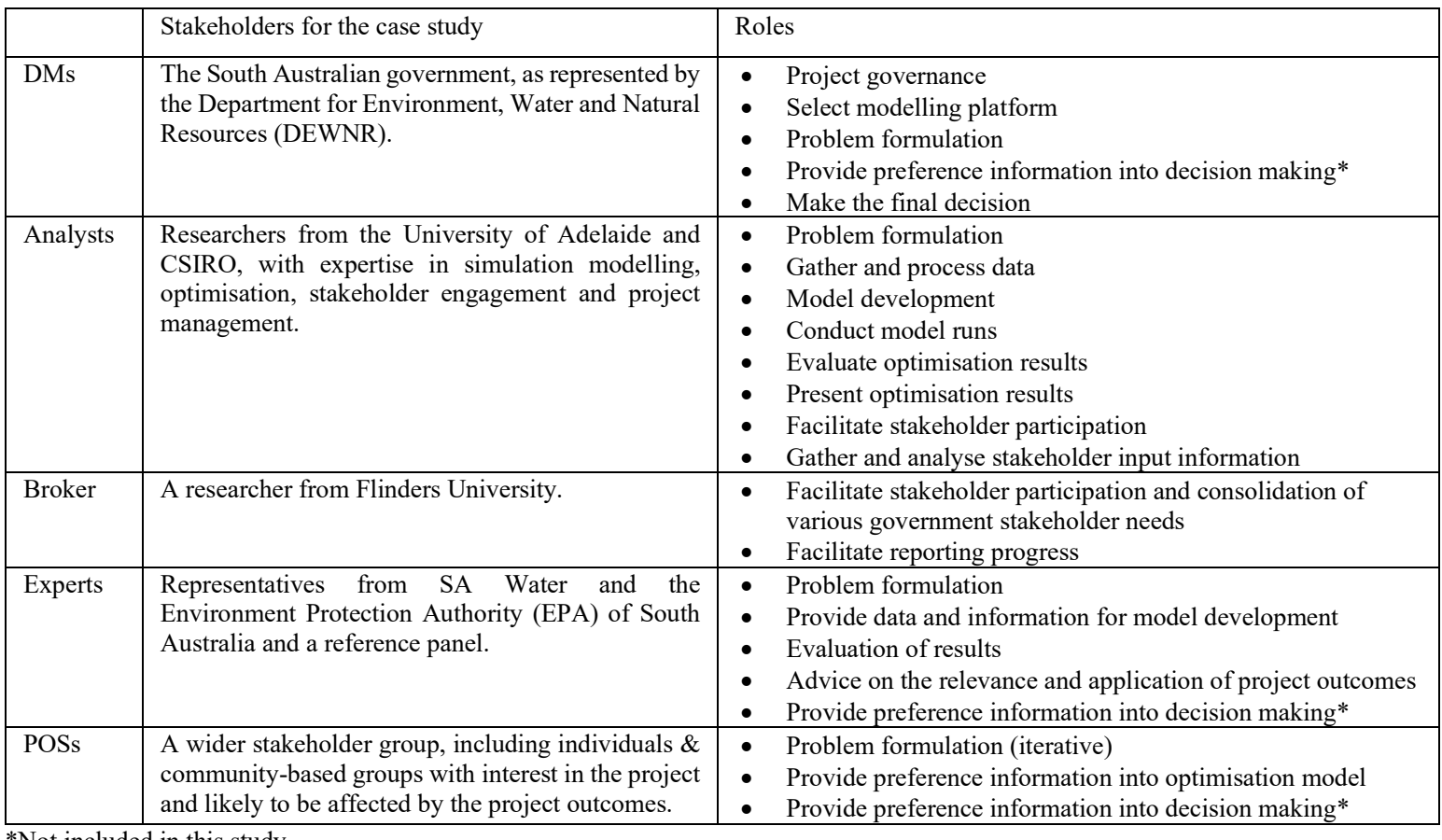

*Not included in this study

\subsubsection{Stage I: Problem formulation}

\section{Step 1: Identify the overall goal.}

Stakeholder input in relation to the identification of the overall goal of the project was provided by the principal DM (i.e. the South Australian Government represented by DEWNR), constrained by the research and development road map of the funding agency (i.e. the Goyder Institute for Water Research).

\section{Step 2: Formulate the problem.}

Stakeholder input in relation to the conceptual formulation of the problem was via the principal DM. This was achieved through meetings between the DM, the principal Analysts, and the funding agency, and facilitated by the Broker. In latter stages of finalising the project design, the agency charged with managing Adelaide's potable water supply, SA Water, joined the project team and provided input.

The outcome of the above stakeholder consultation process was the identification of the main issues and physical system to be addressed in this study, which were to solve water supply issues in Metropolitan Adelaide using Integrated Urban Water Management (IUWM) principles for both 2013 and 2050 conditions under projected climate change. More specifically, the scope of the study was to identify the most cost and energyeffective and environmentally sustainable portfolio of water sources that is also socially acceptable to meet the current and future water demand of Metropolitan Adelaide.

\section{Step 3: Identify objectives, decision variables and constraints.}

The first iteration in identifying the objectives and constraints was via a workshop with the Analysts, Experts (reference panel) and POSs, facilitated by the Broker. The aim of the workshop was to define the project vision, objectives and constraints. A single vision was agreed to during the workshop, which was "Water resource options that provide a long term, secure and sustainable water supply that supports a liveable, economical and healthy city of which the people are proud". Underpinning this vision were 4 objectives: (1) develop a fit-forpurpose system that is flexible and minimises restrictions; (2) minimise lifecycle costs and maximise lifecycle benefits; (3) consider externalities such as flood risk, amenity, community benefit, biodiversity, 
implementability, and governance constraints (efficiency focus); (4) minimise/improve/manage environmental impacts; and (5) understand the community's preferred source mix components.

The stakeholders had been advised that it was desirable to have a limited number of objectives, and that the Analysts would refine the objectives in order to use the optimisation-based approach selected by the DM. Consequently, in the second iteration, the Analysts refined the objectives identified at the workshop, which were included in the optimisation process to produce preliminary results. The results were evaluated by the Analysts in a third iteration. After three iterations, the final outcome was a manageable number of objectives and constraints that could be incorporated into the optimisation model.

The resulting optimisation objectives included:

- Minimisation of the total life cycle cost.

- Minimisation of the total energy consumption.

- Maximisation of the volumetric reliability of non-potable water supply.

- Minimisation of the weighted discharge of stormwater and wastewater to Gulf St. Vincent.

The objectives were optimised subject to the following constraints:

- The reliability of potable supply calculated on a time basis is greater than or equal to $99.5 \%$.

- $\quad$ Storage targets in the reservoirs in the MLR must be met.

- Adequate flows must be released from storages in the MLR to meet environmental flow requirements.

- Water pumped from the RM is limited by the capacities of the pipelines and the legislated five year water extraction limit (i.e. $650 \mathrm{GL}$ per five years).

In order to identify the most appropriate decision variables, a number of meetings were held among the Analysts and Experts from SA Water to discuss the requirement of the optimisation, and the internal structures and limitations of the modelling and optimisation platform pre-selected by the DM - the Source Integrated Modelling System (www.ewater.com.au). The most appropriate surrogate decision variables that enabled the amount of water taken from different sources to be represented while being able to be included in the system model were defined via two iterations. The process involved a number of test optimisation runs, which enabled a better understanding of the physical system and its responses to be gained. The decision variables were:

- The maximum volume of water that can be pumped from the RM via each pipeline on a monthly basis, subject to extraction limits and pump capacities.

- The maximum volume of water that can be drawn from the ADP on a monthly basis, subject to its maximum capacity.

- $\quad$ The stormwater schemes to be implemented (i.e. which of the possible 25 schemes).

- The increase in recycling capacity of each of the WWTPs, subject to their maximum plant capacities.

- The volume of recycled wastewater going to residential non-potable users as a fraction of the total recycled wastewater supplied from each WWTP.

Step 4: Translate objectives and constraints into measurable criteria.

The translation of the objectives, constraints and decision variables into mathematical form was conducted by the Analysts with expert input from SA Water, and facilitated discussions with stakeholders. Details are given in Maheepala et al. (2014).

\subsubsection{Stage II: Optimisation}

Step 5: Identify alternative options \& Step 6: Evaluate alternative options.

The Source Integrated Modelling System (www.ewater.com.au), which forms part of Australia's National Hydrologic Modelling Platform (NHMP), was preselected by the DM to be used as the modelling and optimisation platform. While the Sourceschematic module was used to develop the water balance model

Table 2. Priority sets of water sources for potable and non-potable use

\begin{tabular}{|c|c|c|}
\hline Priority Set & Priority order for Potable Use ${ }^{1}$ & Priority order for Non-Potable Use ${ }^{1}$ \\
\hline $\begin{array}{l}\# 1 \\
\text { (Cost } \\
\text { efficiency } \\
\text { based) }\end{array}$ & $\begin{array}{l}\text { Mt Lofty Ranges } \\
\text { River Murray } \\
\text { Desalinated Water }\end{array}$ & $\begin{array}{l}\text { Mt Lofty Ranges } \\
\text { River Murray } \\
\text { Harvested stormwater } \\
\text { Reclaimed wastewater } \\
\text { Desalinated water }\end{array}$ \\
\hline $\begin{array}{l}\# 2 \\
\text { (Community } \\
\text { preferences } \\
\text { based) }\end{array}$ & $\begin{array}{l}\text { Mt Lofty Ranges } \\
\text { Desalinated Water } \\
\text { River Murray }\end{array}$ & $\begin{array}{l}\text { Harvested stormwater } \\
\text { Reclaimed wastewater } \\
\text { Mt Lofty Ranges } \\
\text { Desalinated water } \\
\text { River Murray }\end{array}$ \\
\hline
\end{tabular}

Note 1: In order of decreasing priority

to simulate the supply, demand and discharge interaction within the Adelaide system, the Source-optimisation 
module, called Insight, which is based on NSGA-II, was used to conduct the multi-objective optimisation. Similarly, the model itself and climate and demand scenarios were selected by the Analysts with data and input from Experts, by agreement with the DM. Stakeholder preferences to different water sources were included in the optimisation process via built-in penalty functions within the Source-schematic module. Stakeholder input in relation to the priorities of water sources required by Source was via the Experts and the POSs. Based on this input, two priority sets were identified (Table 2): Priority Set \#1 was selected by the Analysts through consultation with representatives from SA Water. It was based primarily on the operating cost of each source and reflected SA Water's desire to keep operating costs to a minimum (i.e. cost efficiency based). Priority Set \#2 was developed from a series of small focus groups established as a primary component of the research into social preferences (i.e. community preferences based). The preference for desalinated water for potable use was predicated on its use of 100\% renewable energy from wind turbines (Leonard et al 2015). These stakeholder preferences had a significant impact on the final optimal solutions obtained.

\section{Step 7: Identify efficient options.}

The efficient solutions obtained for the 2013 scenario using multi-objective optimisation (Figure 2) show that the use of Priority Set 2 (Community preferences based) resulted in slightly lower total energy consumption (especially for the high-cost solutions) and less stormwater and wastewater discharge into the Gulf for the same cost. This is due to the preferred use of harvested stormwater for non-potable uses in place of water from the RM and MLR, which has a lower operating energy. The use of Priority Set 2 results in a slight decrease in volumetric reliability of the non-potable supply, which is still very high $(>99.5 \%)$.

Similar results were obtained for the 2050 scenario. However, under the 2050 scenario, the use of different priority sets did not have a significant impact on either the volumetric reliability of non-potable water supply or the total volume of stormwater and wastewater discharge into the Gulf. This is mainly due to the increased demand in Scenario 2050, which results in a large volume of harvested stormwater and recycled wastewater being required. In addition, the use of Priority Set 2 resulted in the selection of a number of solutions with higher energy consumption. These solutions had relatively higher volumetric reliability of nonpotable water supply compared with solutions with similar costs due to
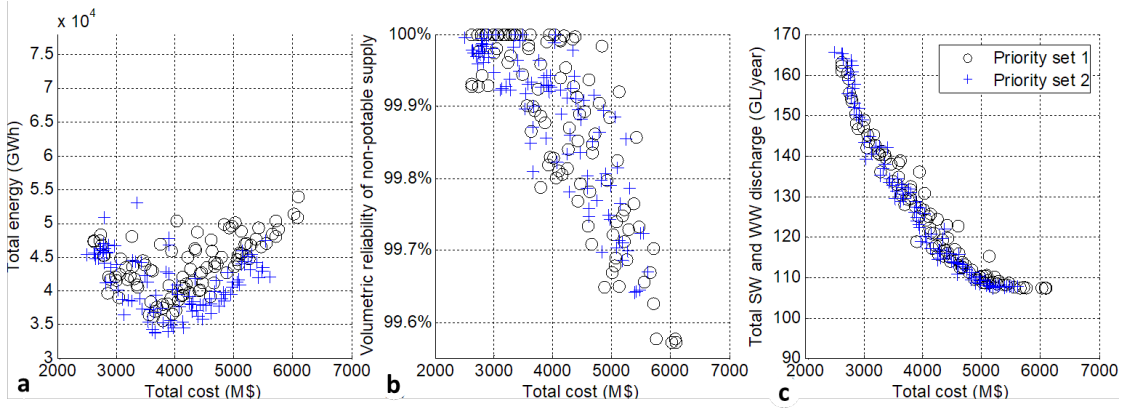

Figure 2. Efficient options for 2013 scenario (Adapted from Wu et al (2016)) their increased supply from the ADP. This indicates that the higher priority given to the ADP in Priority Set 2 encourages the use of desalinated sea water in a more stressed situation, and also demonstrates that the South Australia Government's policy to use the ADP as an emergency source does provide additional water supply security for Adelaide in drought conditions. For interested readers, details of the optimisation results are given in Maheepala et al. (2014) and Wu et al. (2017).

\section{Step 8: Select preferred options.}

In Step 8, the large number of Pareto optimal solutions identified in the multi-objective optimization was reduced by the Analysts to a smaller, more manageable set using compromise programming (CP) (Ballestero, 2007) for presentation to the DMs; resulting in 6 preferred solutions for the 2013 scenario using Priority Set 1 (Cost efficiency based) and 7 preferred solutions using Priority Set 2 (Community preferences based).

\subsubsection{Stage III: Decision analysis}

Step 9: Make the final decision.

The preferred options and the entire problem-solving process were presented by the Analysts to the DMs, Experts and POSs at a concluding workshop, facilitated by the Broker. At this workshop, the framework was endorsed, and results accepted, especially the highlighting of tradeoffs to be considered. While study results did not translate into implementation, the DM (now the Department of Environment and Water) are currently (at time of writing) developing an Urban Water Directions Statement for Adelaide and South Australian towns that optimises the use of all water sources to support growth and greening in a changing climate. 


\section{DISCUSSION AND CONCLUSIONS}

In this paper, a framework for including stakeholder input in real-world water resources optimisation studies is described. The framework is applied to a real-world IUWM problem for Adelaide, South Australia.

This study demonstrates how useful it is to identify relevant stakeholder groups and consider how they are involved in different stages of the process. The results of the case study highlight the significant impact stakeholder input can have on all stages of problem formulation and optimisation. This was particularly the case for the specification of objectives and constraints, the specification of decision variables, the translation of decision variables into measurable criteria and the prioritisation of different water sources during the optimisation process. A number of iterations including different stakeholder groups were required for some of these steps, making use of preliminary optimisation outputs in some instances.

The application of the framework to the Adelaide case study highlighted a number of important lessons: (1) early and regular stakeholder input ensures both real world experience and insight are incorporated and also built trust with those who will implement the decision. (2) including stakeholder input adds cost and time and therefore requires an adequate allowance to be made for this activity in project plans and budgets, (3) the roles of the various stakeholders need to be defined clearly to ensure all parties have the same expectation of the respective roles, (4) there needs to be clear and ongoing communication between stakeholder groups throughout the project, and (5) the use of MOEAs as the optimisation engine together with appropriate stakeholder input provides a combination that is well-suited to solving real-world water resources problems.

\section{ACKNOWLEDGMENTS}

The project was funded through the South Australian Goyder Institute for Water Research and its research and industry partners. Ethics clearance for the engagement with the public focus groups was through the CSIRO's Ethics Committee and in accordance with the National Statement on Ethical Conduct in Human Research. Wenyan Wu acknowledges support from ARC Discovery Early Career Researcher Award (DE210100117).

\section{REFERENCES}

Biswas, A.K., 1976. Systems Approach to Water Management. McGraw-Hill: New York.

Black, D.C., Wallbrink, P.J., Jordan, P.W., 2014. Towards best practice implementation and application of models for analysis of water resources management scenarios. Env Modelling \& Software 52(0) 136-148.

Ballestero, E., 2007. Compromise programming: a utility-based linear-quadratic composite metric from the trade-off between achievement and balanced (noncorner) solutions. Eur. J. Ope. Res. 182, 1369e1382.

Larson, S., Measham, T.G., Williams, L.J., 2010. Remotely engaged? Towards a framework for monitoring the success of stakeholder engagement in remote regions. J. Environ. Plan. Manag. 53(7) 827-845.

Leonard, R., Walton, A. and Farbotko, C. (2015) Using the concept of common pool resources to understand community perceptions of diverse water sources in Adelaide, South Australia. Water Resources Management, 29(5) 1697-1711 DOI 10.1007/s11269-014-0906-6

Maheepala, S., Dandy, G., Marchi, A., Mirza, F., Wu, W., Daly, R., Hewa, G., Neumann, L., Maier, H., 2014. A Decision Support Framework for identifying optimal water supply portfolios: Metropolitan Adelaide Case Study. The Goyder Institute for Water Research: Adelaide.

Maier H.R., Razavi S., Kapelan Z., Matott L.S., Kasprzyk J. and Tolson B.A., 2019. Introductory overview: Optimization using evolutionary algorithms and other metaheuristics, Env Model \& Softw, 114, 195-213.

Marques, J., Cunha, M., Savic, D.A., 2015. Multi-objective optimization of water distribution systems based on a real options approach. Env Modelling \& Software 63 1-13.

Piemonti, A.D., Babbar-Sebens, M., Jane Luzar, E., 2013. Optimizing conservation practices in watersheds: Do community preferences matter? Water Resources Research 49(10) 6425-6449.

Piscopo, A.N., Kasprzyk, J.R., Neupauer, R.M., 2015. An iterative approach to multi-objective engineering design: Optimization of engineered injection and extraction for enhanced groundwater remediation. Env Modelling \& Software, 69:253-261.

Reed, M.S., 2008. Stakeholder participation for environmental management: A literature review. Biological Conservation 141(10) 2417-2431.

Walker, W.E., 2000. Policy analysis: A systematic approach to supporting policymaking in the public sector. Journal of Multi-Criteria Decision Analysis 9(1-3) 11-27.

Wu, W., Dandy, G.C., Maier, H.R., Maheepala, S., Marchi, A. and Mirza, F. (2017) Identification of Optimal Water Supply Portfolios for a Major City, Journal of Water Resources Planning and Management, 143 (9).

Wu W., Maier H.R., Dandy G.C., Leonard R., Bellette K., Cuddy S. and Maheepala S. (2016) Including stakeholder input in formulating and solving real-world optimisation problems: generic framework and case study, Environmental Modelling and Software, 79, 197-213, DOI:10.1016/j.envsoft.2016.02.012. 